\title{
Potential substrates for periphyton enhancement in Carp-SIS polyculture
}

\author{
Sunila Rai ${ }^{1^{*}}$, Kamala Gharti ${ }^{1 * *}$, Madhav Shrestha $^{1 * * *}$, Rahul Ranjan $^{1 * * * *}$, James \\ Diana $^{2 * * * * * *}$ and Hillary Egna ${ }^{3 * * * * * *}$ \\ ${ }^{1}$ Agriculture and Forestry University, Rampur, Chitwan, Nepal \\ ${ }^{2}$ University of Michigan, Ann Arbor, MI 48104-2210, USA \\ ${ }^{3}$ Oregon State University, Corvallis, OR 97331, USA \\ *E-mail: sunilarai10@gmail.com \\ **E-mail: kamalakcnp@yahoo.com \\ ***E-mail: madhavshrestha1954@gmail.com \\ ****E-mail: mail4rahulrnjn@gmail.com \\ *****E-mail: jimd@umich.edu \\ ******E-mail: hillary.egna@oregonstate.edu
}

\begin{abstract}
A field trial was carried out to test performance of four locally available substrates (split bamboo, whole bamboo, banana midrib and plastic bottle) for periphyton enhancement in farmer's ponds at Seri and Nandapur in Nawalparasi district for 7 months. Six carp species were stocked at $15000 \mathrm{fish} / \mathrm{hectare}$ and SIS at unrecorded densities. Carp was fed with rice bran and mustard oil cake at $1.5 \%$ BW while grass carp was fed with grass and banana leaves at $50 \%$ BW. There was no significant effect of substrates on growth and production of carp. Combined NFY was $19 \%$ higher in plastic bottle ponds than control ponds, while NFY of SIS was $50 \%$ higher in banana midrib ponds than control and other substrate ponds. FCR was significantly better $(\mathrm{P}<0.05)$ in split bamboo ponds than control ponds. Banana midrib decayed fast and was replaced 3-4 times during experimental period while plastic bottles performed better in terms of production and profit.
\end{abstract}

Key words: Carp, SIS, Substrate, Periphyton enhancement, Yield

DOI: http://dx.doi.org/10.3126/on.v16i1.21559

Manuscript details: Received: 18.05.2018 / Accepted: 19.07.2018

Citation: Rai, S., K. Gharti, M. Shrestha, R. Ranjan, J. Diana and H. Egna 2018 Potential substrates for periphyton enhancement in Carp-SIS polyculture Our Nature 16(1): 8-7. DOI: http://dx.doi.org/10.3126/on.v16i1.21559

Copyright: ( ) Rai, Gharti, Shrestha, Ranjan, Diana and Egna 2018. Creative Commons Attribution - Non Commercial 4.0 International License.

\section{Introduction}

Carp polyculture in earthen ponds is a wellestablished aquaculture system in Nepal which contributes about $87.5 \%$ of total fish production (Kunwar and Adhikari, 2016). Pond production system is becoming greatly dependent on external resources such as feed and fertilizers for fish production. It has been shown that feed accounts for about $60 \%$ of total fish input cost in commercial fish farming (Bhujel, 2009). Also in small scale aquaculture the ratio of feed cost in total inputs is large. For small scale farmers it is not easy to bear the cost of expensive food ingredients. Providing an alternative means to reduce the feed cost has thus become essential for sustainability of the farming system. In most pond production systems, only a small 
proportion of nutrient input (30\%) is converted into harvestable matter while the rest is lost into sediments, effluent water and atmosphere (Acosta Nassar et al., 1994; Beveridge et al., 1994; Olah et al., 1994). Improving the conversion of nutrients into harvestable matters by enhancing the natural food production may be a suitable solution to the problem of higher cost as well as loss of nutrient inputs. Enhancing the growth of periphyton in pond production system has been proved to be a suitable method to increase the natural food production (Azim et al., 2001a; Rai et al., 2008; Jha et al., 2018).

Many fish in nature as well as in culture relies on periphyton for its food. Indian major carp (Wahab et al., 1999; Ramesh et al., 1999; Rai et al., 2010), tilapia (Hem and Avit, 1994; Shrestha and Knud-Hansen, 1994; Milstein et al., 2009: Jiwyam, 2013), common carp (Rai and Yi, 2012) as well prawn (Udin et al., 2007) prefer periphyton as natural food. Previous study on periphyton based carp polyculture in Nepal showed a promising result with an increase of $24 \%$ in fish yield (Jha et al., 2018). Many researches in periphyton based aquaculture was carried out using different parts of bamboo as substrate in Bangladesh and Nepal (Azim et al., 2001a, b; Azim et al., 2002; Rai et al., 2008; Shirin et al., 2013; Jha et al., 2018). In previous experiment, farmers using bamboo mat as substrate for periphyton enhancement complained that it interfered with partial harvesting of fish. Considering their problem, present experiment was carried out to assess the performance of locally available alternative substrates in farmer's ponds stocked with carp and SIS.

\section{Materials and methods Experimental design}

The experiment was carried out for 210 days from $12^{\text {th }}$ April to $10^{\text {th }}$ November 2017 in 15 ponds of farmers involved in Mishrit Fish Farmer Cooperative at Seri and Nandapur of Nawalparasi district. The average area of experimental pond was $502.9 \pm 68.4 \mathrm{~m}^{2}$ ranging from 163.0 to $1760.0 \mathrm{~m}^{2}$. The experiment was carried out in completely randomized design (CRD). There were five treatments each with three replicates as shown in Table 1.
Table 1. Description of treatments

\begin{tabular}{cll}
\hline Treatment & \multicolumn{1}{c}{ Substrate type } & \multicolumn{1}{c}{ Fish } \\
\hline $\mathrm{T}_{\mathrm{C}}$ & Control (No substrate) & Carp and SIS \\
$\mathrm{T}_{\mathrm{SB}}$ & Split Bamboo & Carp and SIS \\
$\mathrm{T}_{\mathrm{WB}}$ & Whole Bamboo & Carp and SIS \\
$\mathrm{T}_{\mathrm{BM}}$ & Banana Midrib & Carp and SIS \\
$\mathrm{T}_{\mathrm{PB}}$ & Plastic Bottle & Carp and SIS \\
\hline
\end{tabular}

All ponds were drained and dried and lime was applied at the rate of $50 \mathrm{~g} / \mathrm{m}^{2}$. Ponds were then filled with fresh water from boring. Urea and DAP were applied at the rate of $4.7 \mathrm{~g} / \mathrm{m}^{2}$ and 3.5 $\mathrm{g} / \mathrm{m}^{2}$ respectively for plankton growth. After 3 days of fertilization, substrates were installed in all ponds except control ponds.

\section{Substrate preparation and installation}

Altogether 4 different types of substrate such as split bamboo mat, whole bamboo, banana midrib and plastic bottle were installed in substrate ponds to enhance periphyton growth. Substrates covered about $2 \%$ pond surface area in each pond except control ponds. Surface area for different substrates was determined by measuring its dimensions. Split bamboo substrate was prepared by splitting a whole bamboo into slats of $2-5 \mathrm{~cm}$ width and $1 \mathrm{~m}$ length and weaving slats into a mat. Each split bamboo mat was supported with water filled (sink) and empty (float) bottles for their proper vertical position in the water column. Number of split bamboo mats per pond depended on the area of pond. For whole bamboo substrate, bamboo were installed in a manner so that all branches of bamboo lied underneath water and main stem lied about 20-30 $\mathrm{cm}$ below water surface. Banana midrib substrate was also prepared by weaving midribs of banana leaf into a mat similar to that of split bamboo mat. For bottle substrate, empty bottles of soft drinks were filled with water and tied in a ring. The ring was kept floating using empty water bottles on top of the ring. Number of rings per pond depended on area of pond and number of bottles per ring.

All ponds were stocked with six carp species (silver carp Hypophthalmichthys molitrix, bighead carp Aristichthys nobilis, grass carp Ctenopharyngodon idella, common carp 
Cyprinus carpio, rohu Labeo rohita, mrigal Cirrhinus mrigala) at the rate of 15,000/ha and 2 SIS viz. Pothi (Puntius spp.) and Dedhuwa (Esomus danricus) at unrecorded densities. SIS were stocked to ponds by allowing them to enter from water inlet. Stocking of fish was done after 7 days of fertilization. Stocking information of different fish species is given in Table 2 .

Table 2. Stocking density (No./ha) of carp in different treatments

\begin{tabular}{lcc}
\hline Species & $\begin{array}{l}\text { Stocking } \\
\text { number } \\
\text { (No./ha) }\end{array}$ & Stocking (\%) \\
\hline Silver carp & 3000 & 20 \\
Bighead carp & 750 & 5 \\
Common carp & 3000 & 20 \\
Grass carp & 3000 & 20 \\
Rohu & 3750 & 25 \\
Mrigal & 1500 & 10 \\
\hline Total Carp & 15000 & 100 \\
\hline
\end{tabular}

\section{Feeding and fertilization}

Carp were fed with freshly made dough of mustard oil cake and rice bran (1:1). Feed was provided in a traditional bamboo tray placed in each pond every morning at 9-10 am. Feeding rate was $1.5 \%$ BW. Grass carp was fed with grass and banana leaves at $50 \% \mathrm{BW}$. Urea and DAP was applied fortnightly at rates of $9.4 \mathrm{~g} / \mathrm{m}^{2}$ and $7.2 \mathrm{~g} / \mathrm{m}^{2}$ respectively in all ponds to enhance periphyton and phytoplankton population.

\section{Water quality and Periphyton analysis}

Temperature, DO, $\mathrm{pH}$ and Sechhi disk visibility of ponds were monitored at 7-9 am in situ monthly. Periphyton samples from four different types of substrate were taken from the pond and analyzed three times, in the beginning, middle and end of the trial at laboratory of Fisheries Program in AFU. Two samples were taken from each substrate; first sample was taken for periphyton biomass analysis while second sample was taken to determine periphyton abundance. Samples were collected from $1 \mathrm{~cm}^{2}$ area of substrate by scrapping periphyton from the surface using a sharp scalpel.

(a) Dry matter, ash free dry matter, and ash content were determined following APHA (1980) using following formulae.

$$
\text { Dry Matter }=W_{2}-W_{1}
$$

$$
\begin{aligned}
& \text { Ash Content }(\%)=\frac{W_{3}-W_{1}}{W_{2}-W_{1}} * 100 \\
& \text { Ash Free Dry Matter }=W_{2}-W_{3}
\end{aligned}
$$

Where,

$\mathrm{W}_{1}=$ Weight of Aluminum foil and periphyton sample

$\mathrm{W}_{2}=$ Weight of Aluminum foil and periphyton sample after drying

$\mathrm{W}_{3}=$ Weight after combustion

(b) Second sample was transferred into a reagent bottle containing 10\% formaldehyde. Periphyton genera were identified following Prescott (1951), Guiry and Guiry (2018), Edmondson (1959) and Pennak (1978). Their abundance was estimated at genus level for each substrate.

\section{Gross margin analysis}

Economic return was calculated using gross margin analysis. Gross margin for each treatment was determined by subtracting total variable cost of treatment from gross income.

A one-way analysis of variance (ANOVA) was used to compare effects of treatments on water quality parameters, periphyton abundance and biomass, and on fish growth and production, followed by Duncan's Multiple Range Test. All statistical analyses were performed using SPSS-v 16.0. Alpha was set at 0.05 for all comparisons. All means are reported with \pm 1 standard error (SE).

\section{Results and discussion}

Water quality in all ponds was within an acceptable range for carp indicating that different types of substrate did not affect water quality (Table 3). There was no significant difference between average temperature, average transparency and average dissolved oxygen (DO) among different treatments.

There were no significant differences in periphyton abundance (Table 4) among different treatments. Split bamboo had insignificantly higher abundance of periphyton among four substrates. Altogether 38 species of phytoplankton and 8 species of zooplankton were recorded from four types of substrates. Split bamboo supported higher diversity of periphyton (41genera) followed by plastic bottle (39 genera), banana midrib (38 genera) and whole bamboo (36 genera). 
Table 3. Water quality parameters in different treatments

\begin{tabular}{lccccc}
\hline & $\mathrm{T}_{\mathrm{C}}$ & $\mathrm{T}_{\mathrm{SB}}$ & $\mathrm{T}_{\mathrm{WB}}$ & $\mathrm{T}_{\mathrm{BM}}$ & $\mathrm{T}_{\mathrm{PB}}$ \\
\hline Temperature $\left({ }^{\circ} \mathrm{C}\right)$ & $29.3 \pm 0.3^{\mathrm{a}}$ & $29.8 \pm 0.7^{\mathrm{a}}$ & $30.0 \pm 0.8^{\mathrm{a}}$ & $30.1 \pm 0.5^{\mathrm{a}}$ & $30.1 \pm 0.5^{\mathrm{a}}$ \\
Transparency $(\mathrm{cm})$ & $24 \pm 3^{\mathrm{a}}$ & $25 \pm 4^{\mathrm{a}}$ & $25 \pm 3^{\mathrm{a}}$ & $22 \pm 3^{\mathrm{a}}$ & $23 \pm 3^{\mathrm{a}}$ \\
DO $(\mathrm{mg} / \mathrm{L})$ & $3.9 \pm 0.7^{\mathrm{a}}$ & $4.6 \pm 0.8^{\mathrm{a}}$ & $4.3 \pm 0.8^{\mathrm{a}}$ & $4.5 \pm 0.5^{\mathrm{a}}$ & $4.3 \pm 0.6^{\mathrm{a}}$ \\
$\mathrm{pH}$ & 8.0 & 8.2 & 8.0 & 7.3 & 8.1 \\
\hline
\end{tabular}

Similar superscripts for values in a row indicate no significant difference among the values.

Table 4. Abundance $\left(\right.$ no. $\left./ \mathrm{cm}^{2}\right)$ of periphyton in different substrates

\begin{tabular}{|c|c|c|c|c|}
\hline \multirow[b]{2}{*}{ Group } & \multicolumn{3}{|c|}{ Treatment } & \multirow[b]{2}{*}{$\mathrm{T}_{\mathrm{PB}}$} \\
\hline & $\mathrm{T}_{\mathrm{SB}}$ & $\mathrm{T}_{\mathrm{WB}}$ & $\mathrm{T}_{\mathrm{BM}}$ & \\
\hline \multicolumn{5}{|l|}{ Phytoplankton } \\
\hline \multicolumn{5}{|l|}{ Bacillariophyceae } \\
\hline Coscinodiscus & $3333 \pm 1735^{\mathrm{a}}$ & $3056 \pm 1547^{\mathrm{a}}$ & $2500 \pm 241^{\mathrm{a}}$ & $1806 \pm 1410^{\mathrm{a}}$ \\
\hline Cyclotella & $6250 \pm 867^{a}$ & $5833 \pm 636^{\mathrm{a}}$ & $8472 \pm 2661^{\mathrm{a}}$ & $4722 \pm 1959^{a}$ \\
\hline Diatoma & $6389 \pm 2074$ & $7778 \pm 3456^{a}$ & $7361 \pm 2074^{a}$ & $6389 \pm 2504^{\mathrm{a}}$ \\
\hline Fragillaria & $694 \pm 367^{\mathrm{a}}$ & $0 \pm 0^{\mathrm{b}}$ & $0 \pm 0^{\mathrm{b}}$ & $278 \pm 278^{\mathrm{ab}}$ \\
\hline Navicula & $10556 \pm 4938^{\mathrm{a}}$ & $6806 \pm 3852^{a}$ & $6111 \pm 2434^{\mathrm{a}}$ & $5694 \pm 2410^{\mathrm{a}}$ \\
\hline Nitzschia & $2500 \pm 1339^{a}$ & $2778 \pm 2778^{a}$ & $2778 \pm 2778^{a}$ & $4167 \pm 2295^{a}$ \\
\hline Surirella & $0 \pm 0^{\mathrm{a}}$ & $0 \pm 0^{\mathrm{a}}$ & $417 \pm 417^{\mathrm{a}}$ & $0 \pm 0^{\mathrm{a}}$ \\
\hline Synedra & $4538 \pm 1502^{\mathrm{a}}$ & $3611 \pm 1602^{\mathrm{a}}$ & $3333 \pm 1667^{a}$ & $3889 \pm 1325^{\mathrm{a}}$ \\
\hline Total Bacillariophyceae & $34306 \pm 12523^{a}$ & $29861 \pm 6974^{\mathrm{a}}$ & $30972 \pm 7500^{\mathrm{a}}$ & $26944 \pm 5198^{a}$ \\
\hline \multicolumn{5}{|l|}{ Chlorophyceae } \\
\hline Actinastrum & $1111 \pm 1111^{\mathrm{a}}$ & $2500 \pm 2500^{\mathrm{a}}$ & $278 \pm 278^{a}$ & $0 \pm 0^{\mathrm{a}}$ \\
\hline Ankistrodesmus & $8889 \pm 1234^{\mathrm{a}}$ & $6667 \pm 1049^{a}$ & $8889 \pm 773^{a}$ & $6944 \pm 1637^{\mathrm{a}}$ \\
\hline Chlamydomonas & $2361 \pm 2361^{\mathrm{a}}$ & $694 \pm 694^{a}$ & $278 \pm 278^{a}$ & $2222 \pm 2222^{\mathrm{a}}$ \\
\hline Characium & $0 \pm 0^{\mathrm{a}}$ & $556 \pm 556^{\mathrm{a}}$ & $556 \pm 556^{\mathrm{a}}$ & $278 \pm 278^{\mathrm{a}}$ \\
\hline Chlorella & $5972 \pm 2989^{a}$ & $9583 \pm 4829^{a}$ & $10556 \pm 3729^{a}$ & $11111 \pm 3546^{a}$ \\
\hline Closterium & $972 \pm 605^{\mathrm{a}}$ & $0 \pm 0^{\mathrm{a}}$ & $0 \pm 0^{\mathrm{a}}$ & $0 \pm 0^{\mathrm{a}}$ \\
\hline Cosmarium & $1806 \pm 1085^{\mathrm{a}}$ & $1528 \pm 972^{\mathrm{a}}$ & $556 \pm 556^{\mathrm{a}}$ & $2361 \pm 2361^{\mathrm{a}}$ \\
\hline Crucigenia & $5278 \pm 735^{\mathrm{a}}$ & $3750 \pm 1667^{a}$ & $5694 \pm 3046^{a}$ & $3889 \pm 2286^{\mathrm{a}}$ \\
\hline Gonatozygon & $2639 \pm 1869^{a}$ & $0 \pm 0^{\mathrm{a}}$ & $1528 \pm 1528^{a}$ & $2500 \pm 2500^{\mathrm{a}}$ \\
\hline Mougeotia & $1806 \pm 1085^{\mathrm{a}}$ & $1250 \pm 1250^{\mathrm{a}}$ & $833 \pm 833^{\mathrm{a}}$ & $3056 \pm 1547^{\mathrm{a}}$ \\
\hline Oocystis & $0 \pm 0^{\mathrm{a}}$ & $0 \pm 0^{\mathrm{a}}$ & $0 \pm 0^{\mathrm{a}}$ & $417 \pm 417^{\mathrm{a}}$ \\
\hline Pediastrum & $6528 \pm 2504^{a}$ & $4861 \pm 2572^{a}$ & $10139 \pm 5576^{a}$ & $6667 \pm 1684^{\mathrm{a}}$ \\
\hline Scenedesmus & $2222 \pm 1137^{a}$ & $1667 \pm 962^{a}$ & $3750 \pm 833^{a}$ & $1667 \pm 1667^{\mathrm{a}}$ \\
\hline Selenastrum & $417 \pm 417^{\mathrm{a}}$ & $694 \pm 694^{\mathrm{a}}$ & $0 \pm 0^{\mathrm{a}}$ & $694 \pm 694^{\mathrm{a}}$ \\
\hline Staurastrum & $556 \pm 556^{\mathrm{a}}$ & $694 \pm 694^{\mathrm{a}}$ & $833 \pm 481^{\mathrm{a}}$ & $278 \pm 278^{a}$ \\
\hline Tetreedron & $833 \pm 833^{a}$ & $1250 \pm 1250^{\mathrm{a}}$ & $3056 \pm 3056^{a}$ & $0 \pm 0^{\mathrm{a}}$ \\
\hline Tetraspora & $0 \pm 0^{\mathrm{a}}$ & $0 \pm 0^{\mathrm{a}}$ & $1389 \pm 1389^{a}$ & $694 \pm 694^{\mathrm{a}}$ \\
\hline Ulothrix & $972 \pm 972^{\mathrm{a}}$ & $0 \pm 0^{\mathrm{a}}$ & $0 \pm 0^{\mathrm{a}}$ & $1111 \pm 1111^{\mathrm{a}}$ \\
\hline Volvox & $0 \pm 0^{\mathrm{a}}$ & $0 \pm 0^{\mathrm{a}}$ & $0 \pm 0^{\mathrm{a}}$ & $1111 \pm 1111^{\mathrm{a}}$ \\
\hline Oedogonium & $25972 \pm 17783^{a}$ & $10139 \pm 6590^{a}$ & $10417 \pm 5320^{\mathrm{a}}$ & $20556 \pm 1038^{a}$ \\
\hline Pithophora & $2083 \pm 2083^{a}$ & $0 \pm 0^{\mathrm{a}}$ & $0 \pm 0^{\mathrm{a}}$ & $0 \pm 0^{\mathrm{a}}$ \\
\hline Uronema & $2222 \pm 2222^{\mathrm{a}}$ & $0 \pm 0^{\mathrm{a}}$ & $972 \pm 972^{\mathrm{a}}$ & $0 \pm 0^{\mathrm{a}}$ \\
\hline Total Chlorophyceae & $72638 \pm 6943^{a}$ & $45833 \pm 3971^{\mathrm{a}}$ & $59722 \pm 4968^{\mathrm{a}}$ & $65556 \pm 2022^{a}$ \\
\hline \multicolumn{5}{|l|}{ Cyanophyceae } \\
\hline Anabaena & $6111 \pm 4654^{\mathrm{a}}$ & $5000 \pm 2927^{\mathrm{a}}$ & $3056 \pm 2457^{\mathrm{a}}$ & $5000 \pm 3960^{\mathrm{a}}$ \\
\hline Chroococcus & $3611 \pm 2819^{a}$ & $2917 \pm 2917^{\mathrm{a}}$ & $6111 \pm 6111^{\mathrm{a}}$ & $4861 \pm 2457^{\mathrm{a}}$ \\
\hline Merismopedia & $3194 \pm 1707^{\mathrm{ab}}$ & $5694 \pm 2183^{a}$ & $3750 \pm 2774^{\mathrm{ab}}$ & $2361 \pm 1450^{\mathrm{b}}$ \\
\hline Microcystis & $2222 \pm 605^{a}$ & $2500 \pm 1735^{\mathrm{a}}$ & $2361 \pm 1187^{a}$ & $556 \pm 556^{\mathrm{a}}$ \\
\hline Oscillatoria & $4444 \pm 1806^{a}$ & $3333 \pm 1463^{a}$ & $4444 \pm 1602^{a}$ & $6111 \pm 1325^{\mathrm{a}}$ \\
\hline Total Cyanophyceae & $19583 \pm 8819^{a}$ & $19444 \pm 9343^{a}$ & $19722 \pm 11056^{\mathrm{a}}$ & $18889 \pm 4728^{\mathrm{a}}$ \\
\hline \multicolumn{5}{|l|}{ Euglenophyceae } \\
\hline Euglena & $8333 \pm 1869^{a}$ & $5417 \pm 636^{\mathrm{a}}$ & $8056 \pm 1869^{a}$ & $7778 \pm 1325^{\mathrm{a}}$ \\
\hline Phacus & $972 \pm 605^{\mathrm{a}}$ & $1111 \pm 605^{a}$ & $1667 \pm 636^{a}$ & $1111 \pm 735^{\mathrm{a}}$ \\
\hline Trachalomonas & $4861 \pm 1368^{a}$ & $5556 \pm 3522^{a}$ & $7500 \pm 962^{\mathrm{a}}$ & $5139 \pm 2102^{\mathrm{a}}$ \\
\hline
\end{tabular}


Rai, Gharti, Shrestha, Ranjan, Diana and Egna / Our Nature (2018), 16 (1): 8-16

\begin{tabular}{|c|c|c|c|c|}
\hline Total Euglenophyceae & $14167 \pm 1502^{\mathrm{a}}$ & $12083 \pm 2546^{\mathrm{a}}$ & $17222 \pm 3266^{\mathrm{a}}$ & $14028 \pm 1187^{\mathrm{a}}$ \\
\hline Other & $30278 \pm 15696^{\mathrm{a}}$ & $10139 \pm 6590^{a}$ & $11389 \pm 5700^{\mathrm{a}}$ & $20556 \pm 10348^{\mathrm{a}}$ \\
\hline Total Phytoplankton & $140694 \pm 35210^{\mathrm{a}}$ & $107222 \pm 31753^{a}$ & $127639 \pm 41629^{a}$ & $125417 \pm 19731^{\mathrm{a}}$ \\
\hline \multicolumn{5}{|l|}{ Zooplankton } \\
\hline \multicolumn{5}{|l|}{ Sarcodina } \\
\hline Difflugia & $4722 \pm 1707^{\mathrm{a}}$ & $2639 \pm 845^{\mathrm{a}}$ & $2361 \pm 1773^{a}$ & $3611 \pm 1822^{a}$ \\
\hline Total Sarcodina & $4722 \pm 1707^{\mathrm{a}}$ & $2639 \pm 845^{\mathrm{a}}$ & $2361 \pm 1773^{\mathrm{a}}$ & $3611 \pm 1822^{\mathrm{a}}$ \\
\hline \multicolumn{5}{|l|}{ Rotifera } \\
\hline Asplanchna & $3056 \pm 911^{\mathrm{a}}$ & $4167 \pm 2295^{a}$ & $2639 \pm 1325^{\mathrm{a}}$ & $3194 \pm 1211^{\mathrm{a}}$ \\
\hline Brachionus & $6111 \pm 1773^{a}$ & $3611 \pm 2312^{\mathrm{ab}}$ & $4722 \pm 2650^{\mathrm{ab}}$ & $2917 \pm 1102^{b}$ \\
\hline Keratella & $417 \pm 417^{\mathrm{a}}$ & $972 \pm 972^{\mathrm{a}}$ & $556 \pm 556^{\mathrm{a}}$ & $278 \pm 278^{\mathrm{a}}$ \\
\hline Lecane & $556 \pm 556^{\mathrm{a}}$ & $556 \pm 556^{\mathrm{a}}$ & $694 \pm 694^{\mathrm{a}}$ & $278 \pm 278^{a}$ \\
\hline Total Rotifera & $10139 \pm 1002^{\mathrm{a}}$ & $9306 \pm 972^{\mathrm{a}}$ & $8611 \pm 1470^{\mathrm{a}}$ & $6667 \pm 636^{\mathrm{a}}$ \\
\hline \multicolumn{5}{|l|}{ Crustacea } \\
\hline Cyclops & $556 \pm 556^{\mathrm{a}}$ & $972 \pm 501^{\mathrm{a}}$ & $556 \pm 556^{\mathrm{a}}$ & $833 \pm 481^{\mathrm{a}}$ \\
\hline Daphnia & $278 \pm 278^{a}$ & $139 \pm 139^{a}$ & $0 \pm 0^{\mathrm{a}}$ & $0 \pm 0^{\mathrm{a}}$ \\
\hline Nauplius & $556 \pm 139^{a}$ & $833 \pm 481^{a}$ & $556 \pm 278^{a}$ & $694 \pm 367^{a}$ \\
\hline Total Crustacea & $972 \pm 972^{\mathrm{a}}$ & $1944 \pm 972^{\mathrm{a}}$ & $1111 \pm 735^{\mathrm{a}}$ & $1528 \pm 845^{\mathrm{a}}$ \\
\hline Total Zooplankton & $15833 \pm 1879^{a}$ & $13889 \pm 911^{\mathrm{a}}$ & $12083 \pm 3014^{a}$ & $11806 \pm 2650^{\mathrm{a}}$ \\
\hline
\end{tabular}

Similar superscripts for values in a row indicate no significant difference among the values.

Periphyton biomass was determined in terms of dry matter, ash and ash-free dry matter (Table 5). There was no significant difference $(p>0.05)$ in dry matter, ash content and ash free dry matter among different types of substrates. There was no significant difference $(\mathrm{p}>0.05)$ in growth and yield of carp and SIS among different treatments except daily weight gain in rohu (Table 6). Daily weight gain (DWG) of rohu in plastic bottle ponds was significantly higher $(p<0.05)$ than in control ponds but the value did not differ with other substrate ponds. Higher DWG of rohu in substrate ponds compared to control ponds can be attributed to its periphyton grazing habit (NFEP, 1997, Rai et al., 2012). Similarly, net yield of carp, combined GFY and combined NFY did not differ among different treatments which can be attributed to similar abundance of periphyton found in substrate ponds. Insignificantly higher combined NFY was observed in substrate ponds compared to control ponds which may be due to insignificantly higher production of rohu and common carp in periphyton enhanced ponds. In plastic bottle ponds, combined NFY was $19 \%$ higher than control ponds. Feed conversion ratio was significantly lower $(p<0.05)$ in split bamboo ponds than control ponds but it was similar to values in other substrate ponds. The reason might be comparatively higher population of periphyton in split bamboo among four substrates and no periphytons in control ponds.
Comparatively higher total carp yield in substrate ponds than control ponds can be attributed to the provision of additional food in terms of periphyton (Miller and Falace, 2000) and bacterial biofilm (Ramesh, 1999). All treatments with periphyton enhancement gave comparatively higher fish yield than the control. Among substrates used, ponds with plastic bottle substrate gave higher fish yield than natural substrates which differed from results obtained in previous work, where natural substrates such as bamboo produced higher yield (van Dam et $a l ., 2002)$. Most likely differences in the surface area of each substrate type, the exposure to sunlight, and the attraction of algae to the substrate surface made each substrate type a unique environment for production of periphyton and the resulting difference in fish production.

Among substrates used, farmers complained about using banana midrib as it has to be replaced 3-4 times during a production cycle. Banana midrib decayed in ponds within 2-3 months which created trouble to farmers. Although banana is easily available from the farm and has multiple uses, replacement effort is important and care should be given on use of it because its decay may cause oxygen depletion in the pond.

There was no significant difference $(p>0.05)$ in feed cost and total variable cost among different treatments (Table 7). Similarly, there was no significant difference $(p>0.05)$ in 
Table 5. Periphyton biomass $\left(\mathrm{g} / \mathrm{cm}^{2}\right)$ in different substrates

\begin{tabular}{lcccc}
\hline & $\mathrm{T}_{\mathrm{SB}}$ & $\mathrm{T}_{\mathrm{WB}}$ & $\mathrm{T}_{\mathrm{BM}}$ & $\mathrm{T}_{\mathrm{PB}}$ \\
\hline Dry matter & $0.0292 \pm 0.0068^{\mathrm{a}}$ & $0.0271 \pm 0.0099^{\mathrm{a}}$ & $0.0432 \pm 0.0123^{\mathrm{a}}$ & $0.0409 \pm 0.0056^{\mathrm{a}}$ \\
Ash content & $0.0205 \pm 0.0061^{\mathrm{a}}$ & $0.0157 \pm 0.0071^{\mathrm{a}}$ & $0.0337 \pm 0.0107^{\mathrm{a}}$ & $0.0313 \pm 0.0044^{\mathrm{a}}$ \\
Ash free dry matter & $0.0087 \pm 0.0010^{\mathrm{a}}$ & $0.0114 \pm 0.0031^{\mathrm{a}}$ & $0.0095 \pm 0.0025^{\mathrm{a}}$ & $0.0095 \pm 0.0012^{\mathrm{a}}$
\end{tabular}

Similar superscripts for values in a row indicate no significant difference among the values.

Table 6. Growth performance of carp and SIS in different treatment (Mean \pm SE)

\begin{tabular}{|c|c|c|c|c|c|}
\hline \multirow{3}{*}{ Parameters } & \multirow{2}{*}{\multicolumn{5}{|c|}{ Treatments }} \\
\hline & & & & & \\
\hline & $\mathrm{T}_{\mathrm{C}}$ & $\mathrm{T}_{\mathrm{SB}}$ & $\mathrm{T}_{\mathrm{WB}}$ & $\mathrm{T}_{\mathrm{BM}}$ & $\mathrm{T}_{\mathrm{PB}}$ \\
\hline \multicolumn{6}{|c|}{ Silver carp } \\
\hline Initial mean weight (g/fish) & $0.7 \pm 0.0^{\mathrm{a}}$ & $0.7 \pm 0.0^{\mathrm{a}}$ & $0.7 \pm 0.0^{\mathrm{a}}$ & $0.7 \pm 0.0^{\mathrm{a}}$ & $0.7 \pm 0.0^{\mathrm{a}}$ \\
\hline Initial total weight $\left(\mathrm{g} / 100 \mathrm{~m}^{2}\right)$ & $21.0 \pm 0.0^{\mathrm{a}}$ & $21.0 \pm 0.0^{\mathrm{a}}$ & $21.0 \pm 0.0^{\mathrm{a}}$ & $21.0 \pm 0.0^{\mathrm{a}}$ & $21.0 \pm 0.0^{\mathrm{a}}$ \\
\hline Final mean weight (g/fish) & $\mathbf{1 3 8 . 4} \pm 27.4^{\mathrm{a}}$ & $172.1 \pm 38.8^{\mathrm{a}}$ & $160.8 \pm 30.1^{\mathrm{a}}$ & $198.1 \pm 32.8^{\mathrm{a}}$ & $183.5 \pm 42.4^{\mathrm{a}}$ \\
\hline Final total weight $\left(\mathrm{kg} / 100 \mathrm{~m}^{2}\right)$ & $3.5 \pm 0.8^{\mathrm{a}}$ & $4.6 \pm 1.1^{\mathrm{a}}$ & $4.0 \pm 0.9^{\mathrm{a}}$ & $4.1 \pm 0.7^{\mathrm{a}}$ & $4.5 \pm 0.7^{\mathrm{a}}$ \\
\hline DWG (g/fish/day) & $0.66 \pm 0.13^{\mathrm{a}}$ & $0.82 \pm 0.18^{\mathrm{a}}$ & $0.76 \pm 0.14^{\mathrm{a}}$ & $0.94 \pm 0.16^{\mathrm{a}}$ & $0.87 \pm 0.20^{\mathrm{a}}$ \\
\hline TWG (kg/pond) & $3.5 \pm 0.8^{\mathrm{a}}$ & $4.6 \pm 1.1^{\mathrm{a}}$ & $4.0 \pm 0.9^{\mathrm{a}}$ & $4.1 \pm 0.7^{\mathrm{a}}$ & $4.5 \pm 0.7^{\mathrm{a}}$ \\
\hline Survival (\%) & $84.5 \pm 7.2^{\mathrm{a}}$ & $88.5 \pm 3.6^{\mathrm{a}}$ & $81.7 \pm 3.6^{\mathrm{a}}$ & $74.1 \pm 17.2^{\mathrm{a}}$ & $85.5 \pm 7.6^{\mathrm{a}}$ \\
\hline Extrapolated GFY (t/ha/yr) & $0.62 \pm 0.14^{\mathrm{a}}$ & $0.80 \pm 0.20^{\mathrm{a}}$ & $0.70 \pm 0.16^{\mathrm{a}}$ & $0.72 \pm 0.13^{\mathrm{a}}$ & $0.79 \pm 0.12^{\mathrm{a}}$ \\
\hline Extrapolated NFY (t/ha/yr) & $0.61 \pm 0.14^{\mathrm{a}}$ & $0.80 \pm 0.20^{\mathrm{a}}$ & $0.69 \pm 0.16^{\mathrm{a}}$ & $0.71 \pm 0.13^{\mathrm{a}}$ & $0.78 \pm 0.12^{\mathrm{a}}$ \\
\hline \multicolumn{6}{|c|}{ Bighead carp } \\
\hline Initial mean weight (g/fish) & $25.6 \pm 0.0^{\mathrm{a}}$ & $25.6 \pm 0.0^{\mathrm{a}}$ & $25.6 \pm 0.0^{\mathrm{a}}$ & $25.6 \pm 0.0^{\mathrm{a}}$ & $25.6 \pm 0.0^{\mathrm{a}}$ \\
\hline Initial total weight $\left(\mathrm{g} / 100 \mathrm{~m}^{2}\right)$ & $194.4 \pm 0.5^{\mathrm{a}}$ & $191.6 \pm 2.0^{\mathrm{a}}$ & $193.5 \pm 0.8^{\mathrm{a}}$ & $194.4 \pm 0.2^{\mathrm{a}}$ & $192.6 \pm 1.3^{\mathrm{a}}$ \\
\hline Final mean weight (g/fish) & $203.8 \pm 52.3^{\mathrm{a}}$ & $165.8 \pm 20.7^{\mathrm{a}}$ & $217.6 \pm 21.9^{\mathrm{a}}$ & $224.5 \pm 32.6^{\mathrm{a}}$ & $272.7 \pm 1.6^{\mathrm{a}}$ \\
\hline Final total weight $\left(\mathrm{kg} / 100 \mathrm{~m}^{2}\right)$ & $1.2 \pm 0.4^{\mathrm{a}}$ & $0.8 \pm 0.1^{\mathrm{a}}$ & $1.2 \pm 0.2^{\mathrm{a}}$ & $1.1 \pm 0.1^{\mathrm{a}}$ & $1.7 \pm 0.2^{\mathrm{a}}$ \\
\hline DWG (g/fish/day) & $0.85 \pm 0.25^{\mathrm{a}}$ & $0.67 \pm 0.10^{\mathrm{a}}$ & $0.91 \pm 0.10^{\mathrm{a}}$ & $0.95 \pm 0.16^{\mathrm{a}}$ & $1.18 \pm 0.01^{\mathrm{a}}$ \\
\hline TWG (kg/pond) & $1.0 \pm 0.4^{\mathrm{a}}$ & $0.6 \pm 0.1^{\mathrm{a}}$ & $1.0 \pm 0.2^{\mathrm{a}}$ & $0.9 \pm 0.1^{\mathrm{a}}$ & $1.5 \pm 0.2^{\mathrm{a}}$ \\
\hline Survival (\%) & $73.8 \pm 4.7^{\mathrm{a}}$ & $67.7 \pm 6.0^{\mathrm{a}}$ & $69.5 \pm 6.5^{\mathrm{a}}$ & $62.7 \pm 4.5^{\mathrm{a}}$ & $83.4 \pm 8.9^{\mathrm{a}}$ \\
\hline Extrapolated GFY (t/ha/yr) & $0.21 \pm 0.07^{\mathrm{a}}$ & $0.14 \pm 0.15^{\mathrm{a}}$ & $0.20 \pm 0.03^{\mathrm{a}}$ & $0.18 \pm 0.02^{\mathrm{a}}$ & $0.30 \pm 0.03^{\mathrm{a}}$ \\
\hline Extrapolated NFY (t/ha/yr) & $0.17 \pm 0.07^{\mathrm{a}}$ & $0.11 \pm 0.12^{\mathrm{a}}$ & $0.17 \pm 0.03^{\mathrm{a}}$ & $0.15 \pm 0.02^{\mathrm{a}}$ & $0.26 \pm 0.03^{\mathrm{a}}$ \\
\hline \multicolumn{6}{|c|}{ Grass carp } \\
\hline Initial mean weight (g/fish) & $0.1 \pm 0.0^{\mathrm{a}}$ & $0.1 \pm 0.0^{\mathrm{a}}$ & $0.1 \pm 0.0^{\mathrm{a}}$ & $0.1 \pm 0.0^{\mathrm{a}}$ & $0.1 \pm 0.0^{\mathrm{a}}$ \\
\hline Initial total weight $\left(\mathrm{g} / 100 \mathrm{~m}^{2}\right)$ & $3.2 \pm 0.0^{\mathrm{a}}$ & $3.2 \pm 0.0^{\mathrm{a}}$ & $3.2 \pm 0.0^{\mathrm{a}}$ & $3.2 \pm 0.0^{\mathrm{a}}$ & $3.2 \pm 0.0^{\mathrm{a}}$ \\
\hline Final mean weight (g/fish) & $234.2 \pm 54.7^{\mathrm{a}}$ & $245.1 \pm 21.8^{\mathrm{a}}$ & $247.0 \pm 45.2^{\mathrm{a}}$ & $286.5 \pm 54.7^{\mathrm{a}}$ & $241.7 \pm 15.3^{\mathrm{a}}$ \\
\hline Final total weight (kg/pond) & $3.9 \pm 1.0^{\mathrm{a}}$ & $3.3 \pm 0.7^{\mathrm{a}}$ & $4.7 \pm 1.5^{\mathrm{ab}}$ & $4.0 \pm 0.4^{\mathrm{b}}$ & $4.1 \pm 0.6^{\mathrm{a}}$ \\
\hline DWG (g/fish/day) & $1.66 \pm 0.26^{\mathrm{a}}$ & $1.84 \pm 0.50^{\mathrm{a}}$ & $1.73 \pm 0.10^{\mathrm{a}}$ & $2.01 \pm 0.25^{\mathrm{a}}$ & $2.11 \pm 0.29^{\mathrm{a}}$ \\
\hline TWG (kg/pond) & $3.9 \pm 1.0^{\mathrm{a}}$ & $3.3 \pm 0.7^{\mathrm{a}}$ & $4.7 \pm 1.5^{\mathrm{a}}$ & $4.0 \pm 0.4^{\mathrm{a}}$ & $4.1 \pm 0.6^{\mathrm{a}}$ \\
\hline Survival (\%) & $55.6 \pm 7.4^{\mathrm{a}}$ & $46.5 \pm 12.3^{\mathrm{a}}$ & $60.7 \pm 10.0^{\mathrm{a}}$ & $52.3 \pm 15.2^{\mathrm{a}}$ & $58.6 \pm 12.1^{\mathrm{a}}$ \\
\hline Extrapolated GFY (t/ha/yr) & $0.68 \pm 0.18^{\mathrm{a}}$ & $0.57 \pm 0.13^{\mathrm{a}}$ & $0.82 \pm 0.27^{\mathrm{a}}$ & $0.70 \pm 0.07^{\mathrm{a}}$ & $0.71 \pm 0.10^{\mathrm{a}}$ \\
\hline Extrapolated NFY (t/ha/yr) & $0.68 \pm 0.18^{\mathrm{a}}$ & $0.57 \pm 0.13^{\mathrm{a}}$ & $0.82 \pm 0.27^{\mathrm{a}}$ & $0.70 \pm 0.07^{\mathrm{a}}$ & $0.71 \pm 0.10^{\mathrm{a}}$ \\
\hline
\end{tabular}


Rai, Gharti, Shrestha, Ranjan, Diana and Egna / Our Nature (2018), 16 (1): 8-16

\begin{tabular}{|c|c|c|c|c|c|}
\hline \multicolumn{6}{|c|}{ Common carp } \\
\hline & $\mathrm{T}_{1}$ & $\mathrm{~T}_{2}$ & $\mathrm{~T}_{3}$ & $\mathrm{~T}_{4}$ & \\
\hline Initial mean weight ( $\mathrm{g} /$ fish) & $4.5 \pm 0.0^{\mathrm{a}}$ & $4.5 \pm 0.0^{\mathrm{a}}$ & $4.5 \pm 0.0^{\mathrm{a}}$ & $4.5 \pm 0.0^{\mathrm{a}}$ & $4.5 \pm 0.0^{\mathrm{a}}$ \\
\hline Initial total weight $\left(\mathrm{g} / 100 \mathrm{~m}^{2}\right)$ & $135.1 \pm 0.1^{\mathrm{a}}$ & $135.1 \pm 0.1^{\mathrm{a}}$ & $134.8 \pm 0.1^{\mathrm{a}}$ & $135.3 \pm 0.2^{\mathrm{a}}$ & $135.0 \pm 0.2^{\mathrm{a}}$ \\
\hline Final mean weight (g/fish) & $353.1 \pm 55.6^{\mathrm{a}}$ & $391.5 \pm 105.3^{\mathrm{a}}$ & $368.7 \pm 21.6^{\mathrm{a}}$ & $426.4 \pm 51.9^{\mathrm{a}}$ & $447.1 \pm 60.6^{\mathrm{a}}$ \\
\hline Final total weight $\left(\mathrm{kg} / 100 \mathrm{~m}^{2}\right)$ & $5.0 \pm 0.9^{\mathrm{a}}$ & $5.0 \pm 1.0^{\mathrm{a}}$ & $5.6 \pm 1.0^{\mathrm{a}}$ & $6.5 \pm 0.3^{\mathrm{a}}$ & $6.3 \pm 1.0^{\mathrm{a}}$ \\
\hline DWG (g/fish/day) & $1.11 \pm 0.26^{\mathrm{a}}$ & $1.17 \pm 0.10^{\mathrm{a}}$ & $1.18 \pm 0.22^{\mathrm{a}}$ & $1.36 \pm 0.26^{\mathrm{a}}$ & $1.15 \pm 0.07^{\mathrm{a}}$ \\
\hline TWG (kg/pond) & $4.8 \pm 0.9^{\mathrm{a}}$ & $4.9 \pm 1.0^{\mathrm{a}}$ & $5.5 \pm 1.0^{\mathrm{a}}$ & $6.4 \pm 0.3^{\mathrm{a}}$ & $6.2 \pm 1.0^{\mathrm{a}}$ \\
\hline Survival (\%) & $46.5 \pm 4.0^{\mathrm{a}}$ & $45.8 \pm 10.1^{\mathrm{a}}$ & $50.2 \pm 6.5^{\mathrm{a}}$ & $52.4 \pm 6.1^{\mathrm{a}}$ & $49.2 \pm 11.1^{\mathrm{a}}$ \\
\hline Extrapolated GFY (t/ha/yr) & $0.86 \pm 0.16^{\mathrm{a}}$ & $0.88 \pm 0.17^{\mathrm{a}}$ & $0.98 \pm 0.17^{\mathrm{a}}$ & $1.14 \pm 0.06^{\mathrm{a}}$ & $1.10 \pm 0.17^{\mathrm{a}}$ \\
\hline Extrapolated NFY (t/ha/yr) & $0.84 \pm 0.16^{\mathrm{a}}$ & $0.85 \pm 0.17^{\mathrm{a}}$ & $0.96 \pm 0.17^{\mathrm{a}}$ & $1.11 \pm 0.06^{\mathrm{a}}$ & $1.08 \pm 0.17^{\mathrm{a}}$ \\
\hline \multicolumn{6}{|c|}{ Rohu } \\
\hline \multicolumn{6}{|c|}{ Treatments } \\
\hline Initial mean weight (g/fish) & $4.3 \pm 0.0^{\mathrm{a}}$ & $4.3 \pm 0.0^{\mathrm{a}}$ & $4.3 \pm 0.0^{\mathrm{a}}$ & $4.3 \pm 0.0^{\mathrm{a}}$ & $4.3 \pm 0.0^{\mathrm{a}}$ \\
\hline Initial total weight $\left(\mathrm{g} / 100 \mathrm{~m}^{2}\right)$ & $161.3 \pm 0.1^{\mathrm{a}}$ & $160.9 \pm 0.3^{\mathrm{a}}$ & $160.9 \pm 0.1^{\mathrm{a}}$ & $160.8 \pm 0.2^{\mathrm{a}}$ & $161.3 \pm 0.1^{\mathrm{a}}$ \\
\hline Final mean weight (g/fish) & $209.5 \pm 15.4^{\mathrm{a}}$ & $198.9 \pm 18.1^{\mathrm{a}}$ & $225.2 \pm 13.7^{\mathrm{a}}$ & $207.1 \pm 13.9^{\mathrm{a}}$ & $316.4 \pm 55.8^{a}$ \\
\hline Final total weight $\left(\mathrm{kg} / 100 \mathrm{~m}^{2}\right)$ & $4.9 \pm 0.5^{\mathrm{a}}$ & $6.4 \pm 0.9^{a}$ & $5.7 \pm 1.1^{\mathrm{a}}$ & $6.6 \pm 0.9^{a}$ & $5.8 \pm 0.8^{\mathrm{a}}$ \\
\hline DWG (g/fish/day) & $0.85 \pm 0.01^{\mathrm{b}}$ & $0.96 \pm 0.05^{\mathrm{ab}}$ & $0.93 \pm 0.05^{\mathrm{ab}}$ & $0.97 \pm 0.04^{\mathrm{ab}}$ & $1.01 \pm 0.03^{\mathrm{a}}$ \\
\hline TWG (kg/pond) & $4.7 \pm 0.5^{\mathrm{a}}$ & $6.2 \pm 0.9^{a}$ & $6.5 \pm 0.9^{a}$ & $9.6 \pm 0.6^{\mathrm{a}}$ & $5.7 \pm 0.8^{\mathrm{a}}$ \\
\hline Survival $(\%)$ & $71.3 \pm 0.9^{\mathrm{a}}$ & $82.2 \pm 7.8^{\mathrm{a}}$ & $74.2 \pm 11.0^{\mathrm{a}}$ & $84.2 \pm 9.7^{\mathrm{a}}$ & $752.1 \pm 9.7^{\mathrm{a}}$ \\
\hline Extrapolated GFY (t/ha/yr) & $0.85 \pm 0.09^{\mathrm{a}}$ & $1.11 \pm 0.06^{\mathrm{a}}$ & $0.98 \pm 0.19^{\mathrm{a}}$ & $1.15 \pm 0.16^{\mathrm{a}}$ & $1.01 \pm 0.14^{\mathrm{a}}$ \\
\hline Extrapolated NFY (t/ha/yr) & $0.83 \pm 0.09^{a}$ & $1.08 \pm 0.16^{\mathrm{a}}$ & $0.96 \pm 0.19^{\mathrm{a}}$ & $1.12 \pm 0.16^{\mathrm{a}}$ & $0.98 \pm 0.14^{\mathrm{a}}$ \\
\hline \multicolumn{6}{|c|}{ Mrigal } \\
\hline Initial mean weight (g/fish) & $4.5 \pm 0.0^{\mathrm{a}}$ & $4.5 \pm 0.0^{\mathrm{a}}$ & $4.5 \pm 0.0^{\mathrm{a}}$ & $4.5 \pm 0.0^{\mathrm{a}}$ & $4.5 \pm 0.0^{\mathrm{a}}$ \\
\hline Initial total weight $\left(\mathrm{g} / 100 \mathrm{~m}^{2}\right)$ & $67.4 \pm 0.3^{\mathrm{a}}$ & $66.9 \pm 0.4^{\mathrm{a}}$ & $67.5 \pm 0.2^{\mathrm{a}}$ & $67.1 \pm 0.1^{\mathrm{a}}$ & $67.6 \pm 0.1^{\mathrm{a}}$ \\
\hline Final mean weight (g/fish) & $195.2 \pm 35.0^{\mathrm{a}}$ & $191.7 \pm 25.8^{\mathrm{a}}$ & $172.3 .8 \pm 23.6^{\mathrm{a}}$ & $171.8 \pm 36.6^{\mathrm{a}}$ & $197.0 \pm 20.4^{\mathrm{a}}$ \\
\hline Final total weight $\left(\mathrm{kg} / 100 \mathrm{~m}^{2}\right)$ & $2.4 \pm 0.4^{\mathrm{a}}$ & $2.1 \pm 0.4^{\mathrm{a}}$ & $2.1 \pm 0.5^{\mathrm{a}}$ & $2.3 \pm 0.5^{\mathrm{a}}$ & $2.4 \pm 0.5^{\mathrm{a}}$ \\
\hline DWG (g/fish/day) & $0.91 \pm 0.17^{\mathrm{a}}$ & $0.89 \pm 0.12^{\mathrm{a}}$ & $0.80 \pm 0.11^{\mathrm{a}}$ & $0.80 \pm 0.17^{\mathrm{a}}$ & $0.92 \pm 0.10^{\mathrm{a}}$ \\
\hline TWG (kg/pond) & $2.3 \pm 0.4^{\mathrm{a}}$ & $2.0 \pm 0.4^{\mathrm{a}}$ & $2.1 \pm 0.5^{\mathrm{a}}$ & $2.2 \pm 0.5^{\mathrm{a}}$ & $2.4 \pm 0.5^{\mathrm{a}}$ \\
\hline Survival (\%) & $81.6 \pm 3.6^{\mathrm{a}}$ & $71.9 \pm 6.6^{\mathrm{a}}$ & $80.3 \pm 9.4^{\mathrm{a}}$ & $87.8 \pm 4.2^{\mathrm{a}}$ & $80.3 \pm 9.6^{\mathrm{a}}$ \\
\hline Extrapolated GFY (t/ha/yr) & $0.41 \pm 0.06^{\mathrm{a}}$ & $0.36 \pm 0.08^{\mathrm{a}}$ & $0.37 \pm 0.08^{\mathrm{a}}$ & $0.39 \pm 0.09^{\mathrm{a}}$ & $0.42 \pm 0.09^{\mathrm{a}}$ \\
\hline Extrapolated NFY (t/ha/yr) & $0.40 \pm 0.06^{\mathrm{a}}$ & $0.35 \pm 0.08^{a}$ & $0.36 \pm 0.08^{\mathrm{a}}$ & $0.38 \pm 0.09^{\mathrm{a}}$ & $0.41 \pm 0.09^{\mathrm{a}}$ \\
\hline NFY carp only (t/ha/yr) & $3.63 \pm 0.49^{a}$ & $3.87 \pm 2.78^{\mathrm{a}}$ & $4.05 \pm 0.74^{\mathrm{a}}$ & $4.28 \pm 0.97^{\mathrm{a}}$ & $4.34 \pm 3.75^{\mathrm{a}}$ \\
\hline NFY of SIS only (t/ha/yr) & $0.07 \pm 0.47^{\mathrm{a}}$ & $0.07 \pm 2.78^{a}$ & $0.1 \pm 0.02^{\mathrm{a}}$ & $0.1 \pm 0.97^{\mathrm{ab}}$ & $0.08 \pm 3.75^{\mathrm{a}}$ \\
\hline Combined GFY (t/ha/yr) & $3.70 \pm 0.4^{\mathrm{a}}$ & $3.93 \pm 0.28^{\mathrm{a}}$ & $4.15 \pm 0.74^{\mathrm{a}}$ & $4.38 \pm 0.10^{\mathrm{a}}$ & $4.41 \pm 0.37^{\mathrm{a}}$ \\
\hline Combined NFY (t/ha/yr) & $3.59 \pm 0.49^{a}$ & $3.82 \pm 0.28^{\mathrm{a}}$ & $4.03 \pm 0.74^{\mathrm{a}}$ & $4.27 \pm 0.10^{\mathrm{a}}$ & $4.30 \pm 0.37^{\mathrm{a}}$ \\
\hline Feed conversion ratio (FCR) & $2.0 \pm 0.0^{\mathrm{b}}$ & $1.5 \pm 0.1^{\mathrm{a}}$ & $1.7 \pm 0.2^{\mathrm{ab}}$ & $1.8 \pm 0.1^{\mathrm{ab}}$ & $1.9 \pm 0.2^{\mathrm{ab}}$ \\
\hline
\end{tabular}

Similar superscripts for values in a row indicate no significant difference among the values. 
Table 7. Gross margin (Rs/100 $\mathrm{m}^{2}$ pond) analysis for each treatment after 210 days

\begin{tabular}{|c|c|c|c|c|c|}
\hline & $\mathrm{T}_{\mathrm{C}}$ & $\mathrm{T}_{\mathrm{SB}}$ & $\mathrm{T}_{\mathrm{WB}}$ & $\mathrm{T}_{\mathrm{BM}}$ & $\mathrm{T}_{\mathrm{PB}}$ \\
\hline \multicolumn{6}{|l|}{ Cost } \\
\hline Carp fingerlings & $488 \pm 0$ & $487 \pm 1$ & $487 \pm 0$ & $488 \pm 0$ & $488 \pm 0$ \\
\hline Lime & $72 \pm 0$ & $72 \pm 0$ & $72 \pm 0$ & $72 \pm 0$ & $72 \pm 0$ \\
\hline Urea & $164 \pm 1$ & $166 \pm 0$ & $166 \pm 0$ & $165 \pm 1$ & $166 \pm 0$ \\
\hline DAP & $329 \pm 1$ & $333 \pm 2$ & $330 \pm 1$ & $331 \pm 1$ & $245 \pm 89$ \\
\hline Feed & $1368 \pm 192^{a}$ & $1066 \pm 75^{\mathrm{a}}$ & $1306 \pm 288^{a}$ & $1452 \pm 117^{\mathrm{a}}$ & $1489 \pm 25^{\mathrm{a}}$ \\
\hline Total Variable Cost & $2422 \pm 193^{\mathrm{a}}$ & $2124 \pm 74^{\mathrm{a}}$ & $2362 \pm 287^{a}$ & $2509 \pm 115^{\mathrm{a}}$ & $2460 \pm 111^{\mathrm{a}}$ \\
\hline \multicolumn{6}{|l|}{ Return } \\
\hline Carp & $6258 \pm 846^{a}$ & $6678 \pm 461^{\mathrm{a}}$ & $6993 \pm 1281^{a}$ & $7386 \pm 206^{a}$ & $7485 \pm 636^{\mathrm{a}}$ \\
\hline SIS & $83 \pm 0^{\mathrm{a}}$ & $75 \pm 17^{\mathrm{a}}$ & $109 \pm 26^{a}$ & $115 \pm 27^{\mathrm{a}}$ & $87 \pm 13^{\mathrm{a}}$ \\
\hline Gross Return & $6342 \pm 846^{a}$ & $6753 \pm 473^{\mathrm{a}}$ & $7102 \pm 1283^{a}$ & $7501 \pm 180^{\mathrm{a}}$ & $7572 \pm 643^{\mathrm{a}}$ \\
\hline Gross Margin & $3920 \pm 655^{\mathrm{a}}$ & $4630 \pm 440^{\mathrm{a}}$ & $4741 \pm 1062^{\mathrm{a}}$ & $4992 \pm 164^{\mathrm{a}}$ & $5111 \pm 749^{\mathrm{a}}$ \\
\hline
\end{tabular}

Similar superscripts for values in a row indicate no significant difference among the values.

return from carp and SIS, gross return and gross margin among different treatments. Equal yield of carp and SIS in control and treatment ponds resulted same return and gross margin among ponds. Although considerably higher gross return and gross margin was found in substrate ponds compared to control ponds but values were statistically similar.

\section{Conclusion}

Farming carp in earthen ponds with small indigenous species (SIS) is a sustainable fish production system for small scale farmers in Nepal. It provides both family nutrition and income from consuming more micro-nutrient rich SIS and selling surplus carp, respectively. Adding locally available substrates to the carpSIS ponds enhances periphyton production which in turn increases growth and yield of carp and reduces feed cost. Adding substrates to the ponds also discourage poaching which is a common problem among small scale farms where ponds are not guarded. Among substrates used in the present trial split bamboo, whole bamboo and plastic bottles are more durable and have potential for periphyton enhancement.

\section{Acknowledgements}

The authors would like to thank Ram Bhajan Mandal for his help to analyze periphytons at laboratory and to all farmers involved in this experiment for their help during experimental period. This research was funded by AquaFish Innovation Lab, Oregon State University, Corvallis, Oregon, USA.

\section{References}

Acosta Nassar, M.V., J. M. Morell and J. R.
Corredor 1994. The nitrogen budget of atropical semi-intansive fresh water fish culture pond. Journal of the WorldAquaculture Society 25(2): 261 - 270.

APHA. 1980. Standard Methods for the Examination of Water and Wastewater. $15^{\text {th }}$ edn. American Public Health Association, American Water Work Association and Water Pollution Control Federation, Washington, DC, USA.

Azim, M.E., M.A. Wahab, A.A. van Dam, M.C.M. Beveridge, A. Milstein and M.C.J. Verdegem 2001a. Optimization of fertilization rate for maximizing periphyton production on artificial substrates and the implications for periphytonbased aquaculture. Aquaculture Research 32: 749-760.

Azim, M.E., M.A. Wahab, A.A. van Dam, M.C.M. Beveridge, E.A. Huisman and M.C.J. Verdegem 2001b. Optimization of stocking ratios of two Indian major carps, Rohu (Labeo rohita Ham.) and Catla (Catla catla Ham.) in a periphyton-based aquaculture system. Aquaculture 203: 33-49.

Azim, M.E., M.C.J. Verdegem, H. Khatoon, M.A. Wahab, A.A. van Dam and M.C.M. Beveridge 2002. A comparison of fertilization, feeding and three periphyton substrates for increasing fish production in freshwater pond aquaculture in Bangladesh. Aquaculture 212: 227-243.

Beveridge, M.C.M., M.A. Wahab and S. Dewan 1994. Effects of daily harrowing onpond soil and water nutrient levels and on rohu fingerling production. Prog. Fish Cult. 56: 282- 287.

Bhujel, R.C. 2009. Increasing role and potential of small-scale aquaculture: National and regional perspectives. In Symposium on small-scale aquiculture for increasing resilience of rural livelihoods in Nepal (pp. 2). Kathmandu, 
Nepal.

Edmondson, W.T. 1959. Fresh water biology. Edward and Hipple (Eds.), $2^{\text {nd }}$ edn. John John Willy and Sons Inc, New York. pp 95-189.

Guiry, M.D. and G.M. Guiry 2018. Algae Base. World-wide electronic publication, National. University of Ireland, Galway. http://www.algaebase.org.

Hem, S. and J.L.B. Avit 1994. First result on 'acadza enclos' as an extensive aquaculture system (West Africa). Bulletin of Marine Science 55: 1038-1049.

Jha, S., S. Rai, M.K. Shrestha, J.S. Diana, R.B. Mandal and H. Egna 2018. Production of periphyton to enhance yield in polyculture ponds with carps and small indigenous species. Aquaculture Reports 9: 74-81.

Jiwyam, W. 2013. Density-dependent growth and production of Nile tilapia (Oreochromis niloticus) fingerlings relative to phytoplankton and periphyton biomass. Our Nature 11(2): 105-115.

Kunwar, P.S. and B. Adhikari 2016. Status and development trend of aquaculture and fisheries in Nepal. Nepalese Journal of Aquaculture and Fisheries 3: In press.

Miller, M.W. and A. Falace 2000. Evaluation methods for trophic resource factor-nutrients, primary production, and associated assemblages. In Artificial Reef Evaluation with Application to Natural Habitats (Seaman Jr., W. Ed.). CRC Press, Florida, USA. pp. 95-126.

Milstein, A., Y. Peretz and S. Harpaz 2009. Culture of organic tilapia to market size in periphyton- based ponds with reduced feed inputs. Aquaculture Research 40: 55-59.

NFEP. 1997. Production enhancement of the Indian major carp, Labeo rohita (hamilton) using bamboo trimmings as a substrate for the growth of periphyton. NFEP Paper No.10. Northwest Fisheries Extension Project, Parbatipur, Dinajpur, Bangladesh.

Olah, L., P. Szabo, A.A. Esteky and S.A. Nezami 1994. Nitrogen processing andretention in Hungarian carp farms. Journal of Applied Ichthyology 10: 215-390.

Pennak, R.W. 1978. Freshwater invertebrate of United States. $2^{\text {nd }}$ Ed., John Wiley and Son, New York. 303p.

Prescott, G.W. 1951. Algae of the western great lakes area. WM.C. Brown Publishers, Dubuque, Iowa, 977p.

Rai, S. and Y. Yi 2012. Nibbling frequency of carps in fed and non-fed periphyton-based aquaculture system. The Israeli Journal of
Aquaculture 64: 818-822.

Rai, S., S.H. Thilsted, M.K. Shrestha, M.A. Wahab and K. Gharti 2012. Improvement of women's livelihoods, income, and nutrition through Carp-SIS-Prawn polyculture in Terai, Nepal. Asian Fisheries Science. Special Issue 25S: 217-225.

Rai, S., Y. Yi, M.A. Wahab, A.N. Bart and J.S. Diana 2008. Comparison of rice straw and bamboo stick substrates in periphyton-based carp polyculture systems. Aquaculture Research 39: 464-473.

Rai, S., Y. Yi, M.A. Wahab, A.N. Bart and J.S. Diana 2010. Comparison of the growth and production of carp in polyculture ponds with supplemental feeding using rice straw and kanchi as substrates. Our Nature 8: 92-105.

Ramesh, M.R., K.M. Shankar, C.V. Mohan and T.J. Varghese 1999. Comparison of three plant substrates for enhancing carp growth through bacterial biofilm. Aquacultural Engineering 19: $119-131$

Shirin, M.F., M.A. Haque, M.A. Hossain, M.R. Haque and M.M. Rahman 2013. Effects of provision of substrate and addition of Mola (Amblypharyngodon mola) on growth and production of Mrigal (Cirrhinus cirrhosus) and Tilapia (Oreochromis niloticus) in polyculture. Bangladesh Research Publications Journal $8(1): 62-68$.

Shrestha, M.K. and C.F. Knud-Hansen 1994. Increasing attached microorganism biomass as a management strategy for Nile tilapia (Oreochromis niloticus) production. Aquaculture Engineering 13: 101-108.

Udin, M.S., A. Farzana, M.K. Fatema, M.E. Azim, M.A. Wahab and M.C.J. Verdegem 2007. Technical evaluation of tilapia (Oreochromis niloticus) monoculture and tilapia- prawn (Macrobrachium rosenbergii) polyculture in earthen ponds with or without substrates for periphyton development. Aquaculture 269: 232-240.

van Dam, A.A., M.C.M. Beveridge, M.E. Azim and M.C.J. Verdegem 2002. The potential of fish production based on periphyton. Reviews in Fish Biology and Fisheries 12: 1-31.

Wahab, M.A., M.A. Mannan, M.A. Huda, M.E. Azim, A.G. Tollervey and M.C.M. Beveridge 1999. Effects of periphyton grown on bamboo substrates on growth and production of Indian major carp, Rohu (Labeo rohita Ham.). Bangladesh Journal of Fish Research 3(1): 110. 